\title{
The Dirac Composite Fermion of the Fractional Quantum Hall Effect
}

\author{
Dam Thanh Son \\ Kadanoff Center for Theoretical Physics, University of Chicago, Chicago, Illinois 60637, USA
}

\begin{abstract}
We review the recently proposed Dirac composite fermion theory of the half-filled Landau level.
\end{abstract}

\section{INTRODUCTION}

The fractional quantum Hall effect (FQHE) was discovered in 1982 [1], only a couple of years following the discovery of the integer quantum Hall effect (IQHE). Being one of the most nontrivial problems of condensed matter physics, the FQHE has attracted the attention of theorists ever since. One of the earliest and most influential works is that by Laughlin [2]. The aim of the current review is to survey recent progress in the understanding of one particular, but important, aspect of the FQHE: the composite fermion (CF) in the half-filled Landau level [3]. In particular, we review the arguments leading to the Dirac CF theory [4].

The quantum Hall problem is attractive for theorists partly because of its very simple starting point: a Hamiltonian describing particles moving on a two-dimensional plane, in a constant magnetic field, and interacting with each other through a two-body potential,

$$
H=\sum_{a=1}^{N} \frac{\left(\mathbf{p}_{a}+\mathbf{A}\left(\mathbf{x}_{a}\right)\right)^{2}}{2 m}+\sum_{\langle a, b\rangle} V\left(\left|\mathbf{x}_{a}-\mathbf{x}_{b}\right|\right) .
$$

Here $\mathbf{A}$ is the gauge potential corresponding to a constant magnetic field. The two-body potential $V$ is normally taken to be the Coulomb potential $V(r)=e^{2} / r$, but many results are valid for a large class of repulsive interactions. The quantum Hall states are characterized by many physical properties, including a quantized Hall resistivity, a vanishing longitudinal resistivity, a bulk energy gap, edge modes, etc. For the purpose of this article, we take the existence of an energy gap to be the defining property of the quantum Hall states. A very simplified summary of the experimental situation is as follows: For certain values of the filling factor, defined as

$$
\nu=\frac{\rho}{B / 2 \pi}
$$


where $\rho$ is the two-dimensional electron density, the system is in one of the quantum Hall states with an energy gap. The values of $\nu$ for which there is a gap are either integers, in which case we have an IQHE, or rational numbers, which correspond to the FQHE.

The existence of a gap for integer $\nu$ can be understood on the basis of the approximation of noninteracting electrons. In a magnetic field $B$, the energy eigenvalues of the one-particle Hamiltonian are organized into Landau levels,

$$
E_{n}=\frac{B}{m}\left(n+\frac{1}{2}\right) .
$$

The degeneracy of each Landau level is $B / 2 \pi$ per unit area. At integer $\nu$, states with $n<\nu$ are filled and those with $n \geq \nu$ are left empty. The system then has a gap equal to the spacing between Landau levels, which is $\omega_{c}=B / m$.

In contrast to the IQHE, the FQHE cannot be understood from the noninteracting limit. For example, when $0<\nu<1$, the lowest Landau level (LLL; $n=0$ ) is partially filled, so the noninteracting Hamiltonian has an exponentially large (in the number of electrons) ground-state degeneracy. The miracle of the FQHE is that for certain rational values of $\nu$, interactions between electrons lead to a gap.

There are two energy scales in the fractional quantum Hall (FQH) problem. The first scale is the cyclotron energy $\omega_{c}=B / m$, whereas the second scale is the interaction energy scale. In the case of the Coulomb interaction, the latter energy scale can be estimated as the potential energy between two neighboring electrons,

$$
\Delta=\frac{e^{2}}{r} \sim e^{2} \sqrt{B}
$$

The FQH problem is usually considered in the limit $\Delta \ll \omega_{c}$. This limit is reached experimentally by taking $B \rightarrow \infty$ at fixed $\nu$; theoretically it is also reached by taking $m \rightarrow 0$ at fixed $B$. When $\Delta \ll \omega_{c}$ one can ignore all Landau levels above the lowest one, and the problem can be reformulated as pertaining to a Hamiltonian which operates only on the LLL,

$$
H=\mathcal{P}_{\mathrm{LLL}} \sum_{\langle a, b\rangle} V\left(\left|\mathbf{x}_{a}-\mathbf{x}_{b}\right|\right),
$$

where $P_{\text {LLL }}$ is the projection to the LLL. This extremely simple Hamiltonian, believed to underlie all the richness of FQH physics, cannot be solved by traditional methods of perturbation theory owing to the lack of a small parameter. In particular, there is only one energy scale - the Coulomb energy scale $\Delta$. The FQH problem is essentially nonperturbative.

\section{FLUX ATTACHMENT}

One of the most productive ideas in the FQH physics has been the idea of the CF. Theoretically, the notion of the $\mathrm{CF}$ itself comes from another concept called flux attachment [5], 
which was applied to the FQHE in a number of groundbreaking works [3,6-8]. Here, I review the standard textbook field theory of the CF, although later on I argue that it needs some nontrivial modification to become the correct low-energy effective theory.

In the FQH case, one "attaches" an even number (in the simplest case, two) of magnetic flux quanta to an electron, transforming it to a new object called the CF. In the field theory language, one starts from a theory of interacting electrons $\psi_{e}$ in $(2+1)$ dimensions in a background magnetic field

$$
\mathcal{L}=i \psi_{e}^{\dagger}\left(\partial_{t}-i A_{0}\right) \psi_{e}-\frac{1}{2 m}\left|\left(\partial_{i}-i A_{i}\right) \psi_{e}\right|^{2}+\ldots
$$

where ... stand for interaction terms, and derives, following a certain formal procedure, a new Lagrangian for the CF $\psi$,

$$
\mathcal{L}=i \psi^{\dagger}\left(\partial_{t}-i A_{0}+i a_{0}\right) \psi-\frac{1}{2 m}\left|\left(\partial_{i}-i A_{i}+i a_{i}\right) \psi\right|^{2}+\frac{1}{2} \frac{1}{4 \pi} \epsilon^{\mu \nu \lambda} a_{\mu} \partial_{\nu} a_{\lambda}+\ldots
$$

The Chern-Simons (CS) term in Eq. (7) encodes the idea of flux attachment. In fact, the equation of motion obtained by differentiating the action with respect to $a_{0}$ reads

$$
2 \psi^{\dagger} \psi=\frac{b}{2 \pi}, \quad b=\boldsymbol{\nabla} \times \mathbf{a},
$$

which means that the magnetic fluxes of the dynamic gauge field $a_{\mu}$ are tied to the location of the CFs, with two units of fluxes per particle.

There are two features of the field theory (7) (which I call the HLR field theory after Halperin, Lee \& Read, who used it to study the half-filled Landau level [3]) that are rather trivial but worth listing here for future reference:

- The number of CFs is the same as the number of electrons. It cannot be otherwise if the $\mathrm{CF}$ results from attaching magnetic fluxes to an electron.

- The action contains a Chern-Simons term for $a_{\mu}$. As demonstrated above, this term encodes in mathematical terms the idea of flux attachment.

In the literature, it is often stressed that transformation from Eq. (6) to Eq. (7) can be done in an exact way (see, e.g., Ref. [8]). Unfortunately that also means that the theory (7) cannot be solved exactly. To make any progress at all, one must start with some approximation scheme, and in every work so far this has been the mean field approximation in which one replaces the dynamical gauge field $a_{\mu}$ by its average value determined from Eq. (8). Because in the Lagrangian (7) the gauge fields $A$ and $a$ enter through the difference $A-a$, and the density of the CFs is the same as the density of the original electrons, the effective average magnetic field acting on $\psi$ is

$$
B_{\text {eff }}=B-\langle b\rangle=B-4 \pi \rho .
$$


Translated to the language of the filling factors,

$$
\nu=\frac{\rho}{B / 2 \pi} \quad \text { and } \quad \nu_{\mathrm{CF}}=\frac{\rho}{B_{\mathrm{eff}} / 2 \pi},
$$

the equation becomes

$$
\nu_{\mathrm{CF}}^{-1}=\nu^{-1}-2 .
$$

In particular, the values $\nu=\frac{n}{2 n+1}$ map to $\nu_{\mathrm{CF}}=n$. In this way, we have mapped the FQH problem for the electrons to the integer quantum Hall (IQH) problem for the CFs, which gives an explanation for the emergence of an energy gap. Experimentally, one finds quite robust quantum Hall plateaus at these values of $\nu$, up to $n \approx 10$.

Another sequence of quantum Hall plateaus are found at $\nu=\frac{n+1}{2 n+1}$. Now $\nu>\frac{1}{2}$ so the

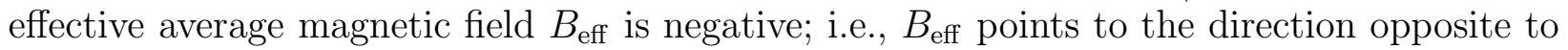
the direction of the original $B$. The CFs still form IQH states, with $n+1$ filled Landau levels $\left[\nu_{\mathrm{CF}}=-(n+1)\right]$. Together, the two series of FQH plateaus at $\nu=\frac{n}{2 n+1}$ and $\nu=\frac{n+1}{2 n+1}$ are called the Jain sequences of plateaus.

One of the most spectacular successes of the CF theory is the prediction of the nature of the $\nu=\frac{1}{2}$ state (the half-filled Landau level) [3]. At this filling fraction, the average effective magnetic field is equal 0 , and the $\mathrm{CF}$ should form a gapless Fermi surface. HLR theory thus predicts that the low-energy excitation is the fermionic quasiparticle near the Fermi surface. There is strong experimental evidence that this is indeed the case [9-11] and that the CF is a real physical object - a quasiparticle near half filling - and not just a mathematical construct.

Despite its astounding success, the quantum field theory (7) has been criticized on various grounds. The criticism leveled most often against the theory (7) is the lack of any information about the projection to the LLL. In particular, the energy gap predicted by the mean-field picture is $B_{\text {eff }} / m$, which, for generic $\nu$, is of order $\omega_{c}$ instead of $\Delta$. To remedy the issue, one has to assume that the energy gap is determined by an effective mass $m_{*}$, postulated to be parametrically $B / \Delta$. In particular, $m_{*}$ is assumed to remain finite in the limit $m \rightarrow 0$.

In fact, there are two parts to the energy scale problems. The first part is to derive, from microscopic calculations, the finite value of $m_{*}$ in the limit $m \rightarrow 0$. This is a difficult problem and we limit ourselves here by noting a few past attempts to address it [12-14]. However, if our goal is only to capture the low-energy physics, i.e., physics at energy scales much smaller than the Fermi energy, then one should simply take the effective mass $m_{*}$ as an input parameter, as in Landau's Fermi liquid theory. The second problem is to make the low-energy effective field theory with $m_{*}$ consistent with the fundamental symmetries of the original theory of electrons with a much smaller mass. Here one expects a relationship similar to the relationship between the effective mass $m_{*}$ and the Fermi-liquid parameter $F_{1}$ in Landau's Fermi liquid theory. This program was pursued in the 1990s and lead to the development of improvements to the HLR theory like the "magnetized modified RPA (MMRPA)" of Simon, Stern and Halperin. (For a field-theoretic interpretation of the MMRPA, see Ref. [15]). In 
principle, the problem can also be solved by using the Newton-Cartan formalism, developed to make explicit the role of Galilean symmetry (see, e.g., Refs. [16-19]).

Interestingly, most recent progress in the physics of the half-filled Landau level has arrived from an attempt to address another problem, historically regarded as less important and subordinate to the energy scale problem: the lack of particle-hole $(\mathrm{PH})$ symmetry.

\section{THE PROBLEM OF PARTICLE-HOLE SYMMETRY}

A system of nonrelativistic particles interacting through a two-body interaction has two discrete symmetries: parity, or spatial reflection $(x \rightarrow x, y \rightarrow-y)$, which we denote as $P$, and time reversal, which we call $T$. In a constant uniform magnetic field both $P$ and $T$ are broken, but $P T$ is preserved. $P T$ transforms the wave function in the following way

$$
\Psi\left(x_{i}, y_{i}\right) \rightarrow \Psi^{\prime}\left(x_{i}, y_{i}\right)=\Psi^{*}\left(x_{i},-y_{i}\right) .
$$

Interestingly, all wave functions that have been proposed, including the Laughlin [2] and Moore-Read [20] wave functions, are invariant under PT.

In the LLL limit $\left(\Delta \ll \omega_{c}\right)$, the projected Hamiltonian (5) has an additional discrete symmetry: the PH symmetry, first considered in Ref. [21]. To define the PH symmetry, one chooses a particular basis of LLL one-particle states $\psi_{k}(x)$. This basis defines the electron creation and annihilation operators $c_{k}^{\dagger}$ and $c_{k}$. The many-body LLL Fock space is obtained by acting products of creation operators on the empty Landau level |empty $\rangle$. PH conjugation, $\Theta$, is defined as an antilinear operator, which maps an empty Landau level to a full one

$$
\left.\Theta: \mid \text { empty }\rangle \rightarrow \mid \text { full }\rangle=\prod_{k=1}^{M} c_{k}^{\dagger} \mid \text { empty }\right\rangle,
$$

where $M$ is the number of orbitals on the LLL. It also maps a creation operator to an annihilation operator and vice versa,

$$
\Theta: c_{k}^{\dagger} \leftrightarrow c_{k} .
$$

One can show that the projected Hamiltonian maps to itself, up to an addition of a chemical potential term,

$$
\Theta: H_{\mathrm{LLL}} \rightarrow H_{\mathrm{LLL}}-\mu_{0} \sum_{k} c_{k}^{\dagger} c_{k}
$$

where $\mu_{0}$ depends on the interaction $V$. This means that for $\mu=\mu_{0} / 2$, the Hamiltonian $H_{\text {LLL }}-\mu N$ maps to itself: At this chemical potential the Hamiltonian is PH symmetric.

Under PH conjugation the filling factor $\nu$ transforms as

$$
\nu \rightarrow 1-\nu \text {. }
$$

In particular $\nu=1 / 2$ maps to itself under $\mathrm{PH}$ conjugation: The half-filled Landau level is at the same time half empty. Furthermore, $\nu=\frac{n}{2 n+1}$ maps to $\nu=\frac{n+1}{2 n+1}$ : the two Jain sequences of quantum Hall plateaus form pairs that map to each other under $\mathrm{PH}$ conjugation: $\nu=1 / 3$ and $\nu=2 / 3, \nu=2 / 5$ and $\nu=3 / 5$, etc. 


\subsection{Status of Particle-Hole Symmetry in the Halperin-Lee-Read Theory}

Let us now ask what are the discrete symmetries of the HLR field theory (7). It is easy to see that there is only one such symmetry, PT. The Chern-Simons theory does not have any discrete symmetry which can be associated with $\mathrm{PH}$ conjugation. This reflects on the asymmetry in the treatment of quantum Hall plateaus: the $\nu=\frac{n}{2 n+1}$ is described by an integer quantum Hall state where the CFs fill $n$ Landau levels, while its PH conjugate $\nu=\frac{n+1}{2 n+1}$ by $n+1$ filled Landau levels.

The Fermi liquid state with $\nu=1 / 2$ presents a particularly baffling problem for $\mathrm{PH}$ symmetry. Naïvely, one expects PH conjugation to map a filled state to an empty state and vice versa. This would mean that the Fermi disk of the CFs, describing the Fermi liquid state, maps to a hollow disk in momentum states: The states with momentum $|\mathbf{k}|>k_{F}$ are filled, and those with $|\mathbf{k}|<k_{F}$ are empty. This is obviously silly.

What makes the PH symmetry problem seem hard is that PH symmetry is not the symmetry of nonrelativistic electrons in a magnetic field [the theory (1)]. It only emerges as the symmetry after taking the lowest Landau level limit [theory (5)]. The PH symmetry of the LLL is not realized as a local operation acting on fields.

Per se, the different treatments of the two Jain's sequences still do not imply that the HLR theory is PH asymmetric. It is logically possible that, despite the appearance to the contrary, the HLR theory would give physical results consistent with PH symmetry. This nontrivial theoretical possibility has been pursued recently. Wang et al. [22] computed the locations of the magnetoroton minima in the Jain states with $\nu=\frac{n}{2 n+1}$ and $\nu=\frac{n+1}{2 n+1}$ at large $n$ using the HLR theory and found that, surprisingly, these locations are symmetric to leading and next-to-leading orders in $1 / n$ (and coincide with the values calculated [23] in the Dirac CF theory described later in this review).

Historically, one of the earliest puzzles posed by PH symmetry on the HLR theory has been recognized in 1997 by Kivelson et al. [24]. When disorders are statistically PH symmetric, PH symmetry implies that at half filling $\sigma_{x y}$ is exactly $\frac{1}{2}\left(e^{2} / h\right)$. In the random phase approximations of the HLR theory, the resistivity tensor of the electrons is directly related to the resistivity of the CFs: $\rho_{x y}=\rho_{x y}^{\mathrm{CF}}+2 h / e^{2}$. The CFs move in an average zero magnetic field, hence one can set $\rho_{x y}^{\mathrm{CF}}=0$, which implies that $\rho_{x y}=2\left(h / e^{2}\right)$. These two results disagree with each other when the longitudinal conductivity $\sigma_{x x}$ (or equivalently, the longitudinal resistivity $\rho_{x x}$ ) is nonzero.

Here too, however, the more recent analysis by Wang et al. finds that the calculation of the Hall conductivity of the CFs may be more subtle than previously thought [22]. The reason is that one can not treat the density of the CFs as a constant in the presence of disorder potential. Detailed calculations by Wang et al. show that, in certain regimes, the

CFs have Hall conductivity $\sigma_{x y}^{\mathrm{CF}}=-\frac{1}{2} e^{2} / h$, exactly the value required so that the Hall conductivity of the electrons is $\frac{1}{2} e^{2} / h$ as dictated by $\mathrm{PH}$ symmetry. 
However, it seems that that the behavior of the quantities considered in Ref. [22] are exceptions rather than the rule. In fact, many other physical observables do not behave in such a fortuitous way. For example, the susceptibility of the two Jain states, $\nu=\frac{n}{2 n+1}$ and

$\nu=\frac{n+1}{2 n+1}$, computed from the MMRPA, differ from each other by a factor of $(n+1)^{2} / n^{2}[25]$. Another example of the violation of PH symmetry in the HLR theory was found in Ref. [15]. In that study, it was shown that $\mathrm{PH}$ symmetry strictly determines the first $q^{2}$ correction to the Hall conductivity in the regime $\omega \gg v_{F} q$,

$$
\sigma_{x y}(\omega, q)=\frac{1}{2}\left[1-\frac{1}{4}\left(q \ell_{B}\right)^{2}\right]
$$

but the MMRPA of the HLR theory fails to get the correct value of $-\frac{1}{4}$ for the $q^{2}$ correction; instead, one would get 0 following the prescription of MMRPA. The discrepancy was traced to the incorrect value of orbital spin assigned to the CF by the flux attachment procedure. One must keep in mind, however, that the calculations in the HLR theory have been performed within a random-phase approximation. The question about the effect of gauge-field fluctuations need to be further investigated [26].

\subsection{Spontaneous Breaking of Particle-Hole Symmetry?}

Another logical possibility is that the Fermi-liquid ground state of the half-filled Landau level spontaneously breaks PH symmetry. This possibility was investigated by Barkeshli et al. [27]. If that is the case, there are two states at $\nu=1 / 2$ : One corresponds to a Fermi surface of "composite particles" and the other to that of "composite holes"; the two states are degenerate with each other in the lowest-Landau level limit. In fact, it is believed that on the second Landau level, the ground state is either a Pfaffian [20] or an anti-Pfaffian state $[28,29]$, which are PH conjugates of each other but are distinct from each other, breaking PH symmetry spontaneously.

However, there is no numerical evidence for this kind of spontaneous $\mathrm{PH}$ symmetry breaking in the Fermi liquid state. In fact, the experimental result of Ref. [30] indicates, at least naïvely, that the $\nu=1 / 2$ Fermi liquid is equally well interpreted as being made out of composite particles or composite holes. There is now strong numerical evidence that the $\nu=1 / 2$ state is $\mathrm{PH}$ symmetric [31].

\section{DIRAC COMPOSITE FERMION}

Having argued against a hidden PH symmetry of the HLR theory and a spontaneous breaking of PH symmetry, we now consider the third, most nontrivial possibility: The low-energy physics of the half-filled Landau level is described by a theory different from the HLR theory. The theory must satisfy PH symmetry but also preserve all successful phenomenological predictions of the HLR theory. The Dirac composite fermion theory, proposed in Ref. [4], 
satisfies these requirements. The essence of the theory is that the $\mathrm{CF}$ does not transform into a composite hole under PH symmetry, but remains a composite particle. Only the momentum of the CF flips sign under PH conjugation,

$$
\Theta: \mathbf{k} \rightarrow-\mathbf{k}
$$

Implicitly, we assume that the Fermi disk of the CFs transform into itself (a filled disk, not a hollow disk).

Equation (18) is usually associated with time reversal. In the theory of Dirac CFs, the $\mathrm{CF}$ is described by a two-component spinor field $\psi$, which transforms under PH conjugation following the formula that is usually identified with time reversal,

$$
\psi \rightarrow i \sigma_{2} \psi
$$

There are several arguments one can put forward to argue that the CF has to be a massless Dirac particle. One hint comes from the CF interpretation of the Jain-sequence states. Recall that one problem with the standard CF picture is that the $\nu=\frac{n}{2 n+1}$ corresponds to the composite fermion filling factor $\nu_{\mathrm{CF}}=n$, whereas $\nu=\frac{n+1}{2 n+1}$ maps to $\nu_{\mathrm{CF}}=n+1$ (ignoring the sign). By constrast, these two states are $\mathrm{PH}$-conjugate pairs and should be described by the same filling factor of the $\mathrm{CF}$ in any $\mathrm{PH}$-symmetric theory. The most naïve way to reconcile these different pictures is to replace the filling factors $\nu_{\mathrm{CF}}=n$ and $\nu_{\mathrm{CF}}=n+1$ with the average value $\nu_{\mathrm{CF}}=n+\frac{1}{2}$. But now we have a problem: We want to map the FQHE in the Jain sequences to the IQHE of the CFs, but is it possible to have an IQH state with half-integer filling factor? Indeed, it is, if the composite fermion is a massless Dirac fermion. Half-integer quantization of the Hall conductivity is a characteristic feature of the Dirac fermion, confirmed in experiments with graphene [32,33].

The second argument in favor of the Dirac nature of the CF relies on a property of the square of the $\mathrm{PH}$ conjugation operator $\Theta^{2}$ [31,34]. It is intuitively clear that applying $\mathrm{PH}$ conjugation twice maps a given state to itself, but there is a nontrivial factor of \pm 1 that one gains by doing so. Consider a generic state on the LLL with $N_{e}$ electrons,

$$
\left.|\psi\rangle=\prod_{i=1}^{N_{e}} c_{k_{i}}^{\dagger} \mid \text { empty }\right\rangle
$$

Then under PH conjugation

$$
\left.\left.\Theta:|\psi\rangle \rightarrow \prod_{i=1}^{N_{e}} c_{k_{i}} \mid \text { full }\right\rangle=\prod_{i=1}^{N_{e}} c_{k_{i}} \prod_{j=1}^{M} c_{j}^{\dagger} \mid \text { empty }\right\rangle .
$$

Applying $\Theta$ again one finds

$$
\left.\left.\Theta^{2}:|\psi\rangle \rightarrow \prod_{i=1}^{N_{e}} c_{k_{i}}^{\dagger} \prod_{j=1}^{M} c_{j} \mid \text { full }\right\rangle=\prod_{i=1}^{N_{e}} c_{k_{i}}^{\dagger} \prod_{j=1}^{M} c_{j} \prod_{k=1}^{M} c_{k}^{\dagger} \mid \text { empty }\right\rangle=(-1)^{M(M-1) / 2}|\psi\rangle .
$$


This relationship is quite easy to interpret when $M$ is an even number: $M=2 N_{\mathrm{CF}}$. Then

$$
\Theta^{2}:|\psi\rangle \rightarrow(-1)^{N_{\mathrm{CF}}}|\psi\rangle
$$

This formula suggests the following interpretation: $N_{\mathrm{CF}}$ is the number of CFs of the state $|\psi\rangle$, and each $\mathrm{CF}$ is associated with a factor of -1 under $\Theta^{2}$. This -1 factor is natural for Dirac fermion.

In order to have a correct $\Theta^{2}$, we have to identify the number of CFs with half the number of orbitals on the LLL: $N_{\mathrm{CF}}=M / 2$, which is independent of the number of electrons $N_{e}$. This contradicts the intuitive picture of flux attachment, in which the CF is obtained by attaching two units of flux quanta to an electron. However, that is expected: In a theory that treats particles and holes in a symmetric way, the number of CFs has to be in general different from the number of electrons, otherwise it would have to be equal to the number of holes as well.

\subsection{The Field Theory of the Dirac Composite Fermion}

The minimal version of the theory of the Dirac CF has the following Lagrangian,

$$
\mathcal{L}=i \psi^{\dagger}\left[\partial_{t}-i a_{0}-v_{\mathrm{F}} \sigma^{i}\left(\partial_{i}-i a_{i}\right)\right] \psi-\frac{1}{4 \pi} \epsilon^{\mu \nu \lambda} A_{\mu} \partial_{\nu} a_{\lambda}+\frac{1}{8 \pi} \epsilon^{\mu \nu \lambda} A_{\mu} \partial_{\nu} A_{\lambda}
$$

where $a_{\mu}$ is a dynamical gauge field. The kinetic term in Eq. (24) contains, instead of the speed of light, a Fermi velocity $v_{\mathrm{F}}$, determined by microscopic physics. The Lagrangian (24) is similar to (7) after the replacement $a \rightarrow a+A$, with two differences. One is the Dirac nature of the CF $\psi$. The other is the absence of the CS term ada in the Lagrangian: Such a term (as also the mass term for $\psi$ ), if present, would disallow any discrete symmetry that could be identified with PH symmetry. Interestingly, each of such modifications to the HLR theory would shift the filling factors of the Jain-sequence plateaus, but together the shifts cancel each other, and the Jain sequences remain unchanged (see below).

Differentiating (24) with respect to $A_{0}$, one obtains the electron density

$$
\rho=\frac{\delta S}{\delta A_{0}}=\frac{B-b}{4 \pi} .
$$

On the other hand, the equation of motion obtained by differentiating the action with respect to $a_{0}$ is

$$
\bar{\psi} \gamma^{0} \psi=\frac{B}{4 \pi}
$$

i.e., the CF density is set by the external magnetic field. Thus, the role of the magnetic field and the density is flipped when one goes from the original electrons to the CFs. This is the salient feature of particle-vortex duality, well known for bosons $[35,36]$ but not, until this example, for fermions. 
If one defines the filling factors of the electron and the $\mathrm{CF}$ as

$$
\nu=\frac{2 \pi \rho}{B} \quad \text { and } \quad \nu_{\mathrm{CF}}=\frac{2 \pi \rho_{\mathrm{CF}}}{b},
$$

respectively, then from Eqs. (25) and (26), we find that they are related by

$$
\nu_{\mathrm{CF}}=-\frac{1}{4\left(\nu-\frac{1}{2}\right)} .
$$

In particular, $\nu=\frac{n}{2 n+1}$ maps to $\nu_{\mathrm{CF}}=n+\frac{1}{2}$, which is the filling factor of an integer quantum Hall state of a Dirac fermion. The PH conjugated state $\nu=\frac{n+1}{2 n+1}$ maps to $\nu_{\mathrm{CF}}=-\left(n+\frac{1}{2}\right)$, which is the same filling factor, but in a magnetic field of the opposite sign, manifesting the PH symmetry.

It should be emphasized that the Dirac nature of the CF does not mean that there is a Dirac cone for the CF. The tip of the cone is at $\mathbf{k}=0$, whereas the CF, as a low-energy mode, exists only near the Fermi surface. The Dirac nature of the CF, strictly speaking, only means that the fermionic quasiparticle has a Berry phase of $\pi$ around the Fermi surface. It is easy to show that such a Berry phase follows from Eqs. (18) and (23). The quasiparticle Berry phase has been identified as a important ingredient of Fermi liquids [37], but the possibility of such a phase for the CF in FQHE has been overlooked in the literature until very recently.

\subsection{Galilean Invariance and Electric Dipole Moment}

It has to be emphasized that the energy scale problem still exists, under a different guise, in the Dirac CF theory. This problem now appears as the problem of the origin of the Fermi velocity of the $\mathrm{CF}$. The scale of the Fermi velocity should be set by the interactions, but, as before, there is no simple way to calculate it from the microscopic theory.

If one takes the Fermi velocity of the $\mathrm{CF}$ as an input parameter, one still has to make sure that the theory of the CF exhibits the symmetry of the original theory, in particular, the Galilean invariance. It is crucial that Galilean invariance is implemented into the effective theory; important properties, like the $q^{4}$ behavior of the susceptibility at small $q$, follow from it. However, the Dirac action is not invariant under Galilean transformation,

$$
\psi(t, \mathbf{x}) \rightarrow \psi^{\prime}(t, \mathbf{x})=\psi(t, \mathbf{x}-\mathbf{v} t) .
$$

To make the Dirac action Galilean invariant, one needs to add an additional term to the effective Lagrangian, replacing

$$
i \psi^{\dagger} \partial_{t} \psi \rightarrow i \psi^{\dagger} \partial_{t} \psi+\frac{i}{2} \frac{\epsilon^{i j} E_{j}}{B}\left(\psi^{\dagger} D_{j} \psi-D_{j} \psi^{\dagger} \psi\right) .
$$

Because the electric field transforms under Galilean transformation as $E_{i} \rightarrow E_{i}-\epsilon^{i j} v_{j} B$, the action is now Galilean invariant. The new term has a very intuitive physical meaning: 
It implies that the CF has an electric dipole moment with respect to the external electromagnetic field, with the dipole moment perpendicular to the momentum: $\mathbf{d}=-\ell_{B}^{2} \mathbf{p} \times \hat{\mathbf{z}}$, realizing an old idea by Read [38,39].

The dipole picture has been revived recently by Wang \& Senthil in the context of the Dirac CF [40]. They suggest that the dipole moment provides a way to understand the origin of the Berry phase of the CF around the Fermi surface. In this picture, the CF is a pair made up of an electron and a correlation hole that form an electric dipole, with the value of the dipole moment perpendicular to the direction of the momentum. When a CF is dragged around the Fermi surface, the dipole rotates and the fermion acquires a Berry phase because of the external magnetic field. A simple calculation shows that the Berry phase is equal to $\pi$ when the CF makes a circle of radius exactly equal to the Fermi momentum in momentum space.

The simplicity of this explanation of the Berry phase may be deceptive, however. If one takes the above dipole picture literally, one finds not only a global Berry phase but also a local Berry curvature that is uniform in momentum space. A massless Dirac fermion, in contrast, has only a global Berry phase but no local Berry curvature. Thus, the connection between the Berry phase and the dipole moment may not be straightforward. Within the low-energy effective theory embodied by Eqs. (24) and (30), the Berry phase and the dipole moment appear as separate ingredients.

\section{CONSEQUENCES OF THE DIRAC COMPOSITE FERMION}

The Dirac CF theory has distinct consequences, which are, in principle, verifiable in experiments and numerical simulations. By construction, it leads to PH-symmetric results where the HLR theory violates PH symmetry. For example, the susceptibilities of the $\nu=\frac{n}{2 n+1}$ and $\nu=\frac{n+1}{2 n+1}$ states are now the same [25].

It is numerical simulations [31] that provide the currently most nontrivial test of the Dirac nature of the CF. The numerical finding is the disappearance, attributable to particlehole symmetry, of the leading $2 k_{F}$ singularity in certain correlation functions. In a Fermi liquid, typical two-point correlation functions exhibit nonanaliticity at twice the Fermi momentum $\left(2 k_{F}\right.$ singularities) due to intermediate states involving a particle and a hole near diametrically opposite points on the Fermi surface. In the case of the $(2+1) d$ massless Dirac fermion, it is well known that two-point correlation functions of time-reversal invariant operators are free from the leading $2 k_{F}$ singularity in a generic two-point correlator. This is caused by the quasiparticle Berry phase $\pi$ around the Fermi surface that forbids the creation, by a time-reversal invariant operator, of a $\mathrm{PH}$ pair where the particle and the hole carry opposite momenta. In the half-filled Landau level, the role of time reversal is played by PH symmetry; therefore to test the Berry phase one should look for the absence of the leading $2 k_{F}$ singularity in correlation functions of a $\mathrm{PH}$ symmetric operator. The electron density operator $\rho=\psi_{e}^{\dagger} \psi_{e}$ is not PH symmetric (the deviation of the density from the mean 
density, $\delta \rho=\rho-\rho_{0}$, flips sign under PH conjugation), but one can easily write down more complicated operators that are PH symmetric, for example, $\delta \rho \nabla^{2} \rho$. In Ref. [31] the leading $2 k_{F}$ singularity in the correlation function of such an operator was shown to disappear when $\mathrm{PH}$ symmetry is made exact (and to reappear when PH symmetry is violated), confirming the Dirac nature of the CF.

There are also predictions about transport that are, strictly speaking, consequences of PH symmetry. If one introduces the conductivities $\sigma_{x x}$ and $\sigma_{x y}$, and the thermoelectric coefficients $\alpha_{x x}$ and $\alpha_{x y}$, so that

$$
\mathbf{j}=\sigma_{x x} \mathbf{E}+\sigma_{x y} \mathbf{E} \times \hat{\mathbf{z}}+\alpha_{x x} \boldsymbol{\nabla} T+\alpha_{x y} \boldsymbol{\nabla} T \times \hat{\mathbf{z}},
$$

then at exact half filling, PH symmetry implies $[4,41]$

$$
\sigma_{x y}=\frac{1}{2} \frac{e^{2}}{h} \quad \text { and } \quad \alpha_{x x}=0 .
$$

A manifestly PH symmetric theory like the Dirac composite fermion theory reproduces these results automatically [41].

In real samples one expects that $\mathrm{PH}$ symmetry is not exact due to the mixing of higher Landau levels. When the breaking of PH symmetry is small, one can expect its effect to be parametrized by a single number: the Berry phase that the CF acquires along the Fermi circle [41].

\subsection{The PH-Pfaffian State}

One consequence of the Dirac CF theory is the existence of a new gapped state at half filling that is PH symmetric. When the CFs undergo Bardeen-Cooper-Schrieffer (BCS) pairing, the resulting state is a gapped quantum Hall state. In the traditional HLR theory, the pairing between fermions has to be in a channel with odd orbital moment to be consistent with Fermi statistics. The Dirac nature of the composite fermion, in contrast, requires the BCS gap to carry an even orbital moment. The simplest pairing channel is [4]

$$
\left\langle\varepsilon^{\alpha \beta} \psi_{\alpha} \psi_{\beta}\right\rangle
$$

and does not break either PT or PH symmetry. The state is the quantum Hall analog of the so-called T-Pfaffian state, a proposed fully gapped state on the interacting surface of topological insulators [42-45]. The PH-Pfaffian state is distinct from the Pfaffian state and the anti-Pfaffian state, which are not $\mathrm{PH}$ symmetric and are the $\mathrm{PH}$ conjugates of each

others. In particular, the shift of the PH-Pfaffian state is equal to 1, whereas the shift of the Pfaffian is 3 and the anti-Pfaffian state carries shift of -1 .

Within the Dirac composite fermion theory, the Pfaffian and anti-Pfaffian states involve pairing with orbital moments \pm 2 , i.e., with order parameters $\left\langle\varepsilon^{\alpha \beta} \psi_{\alpha}\left(\partial_{x} \pm i \partial_{y}\right)^{2} \psi_{\beta}\right\rangle$. The Dirac CF thus provides a symmetric treatment of the Pfaffian and anti-Pfaffian states, with 
the PH-Pfaffian state situating exactly at the midpoint in terms of the orbital moment of the Cooper pair.

In the HLR theory, the orbital moment of the Cooper pair is shifted by one unit compared to the Dirac theory. The Pfaffian state appears as a $p_{x}+i p_{y}$ pairing of the CFs. This interpretation of the Pfaffian state has been known for a long time. The PH-Pfaffian state also corresponds to $p$-wave pairing, but here the orbital moment is of the opposite sign, i.e., $p_{x}-i p_{y}$ pairing. Finally, to get the anti-Pfaffian state from the usual HLR theory, one would pair the CFs in the $\ell=-3$ orbital moment channel. The HLR theory does not explain why the $\ell=1$ and $\ell=-3$ paired states have the same energy.

At this moment, there is no simple particle-hole symmetric wavefunction for the PHPfaffian state (except ones that involve explicit symmetrization of two PH conjugate wavefunctions). However, there seem to exist wavefunctions that are quite close to being $\mathrm{PH}$ symmetric. One simple proposed wave function is [46] (omitting the Gaussian factor)

$$
\Psi\left(z_{1}, \cdots, z_{n}\right)=\operatorname{Pf}\left(\frac{1}{\partial_{z_{i}}-\partial_{z_{j}}}\right) \prod_{\langle i j\rangle}\left(\partial_{z_{i}}-\partial_{z_{j}}\right) \prod_{\langle i j\rangle}\left(z_{i}-z_{j}\right)^{3} .
$$

This wavefunction has a large overlap with its PH conjugate, at least for relatively small number of particles.

It is not at all clear if the PH-Pfaffian state can be realized with any two-body interactions. Numerical simulations either show a Fermi liquid state or a PH-breaking gapped state [31]. On the other hand, Ref. [47] argue that the PH-Pfaffian state may be stabilized by the mixing with higher Landau levels and impurities (note that the topological order of the PHPfaffian does not require PH symmetry to exist) and it may present a viable alternative for the $\nu=5 / 2$ plateau.

\section{UNDERSTANDING THE ORIGIN OF THE DIRAC COMPOSITE FERMION}

As described above, much theoretical and numerical evidence implies that the quasiparticle of the $\nu=1 / 2$ state has to be a Dirac CF. This means, in particular, that the old, intuitive picture of a $\mathrm{CF}$ as an electron with two attached flux quanta (or rather, an electron as a CF with two attached flux quanta) cannot be literally correct.

It is perhaps fair to say that at this moment there is no completely satisfactory derivation of the Dirac CF theory from the microscopic theory of fermions on the LLL. An attempt to embed the Girvin-MacDonald-Platzman algebra into that of Dirac CF was made in Ref. [48]. The role of the gauge field, however, is not clear in this mapping.

Most other attempts to derive the theory of Dirac CFs are actually aimed at deriving a zero magnetic field version: the duality between the free Dirac fermion, defined by the action

$$
\mathcal{L}_{A}=i \bar{\Psi} \gamma^{\mu}\left(\partial_{\mu}-i A_{\mu}\right) \Psi
$$


and a theory of a fermion coupled to an gauge field which we call parity-invariant $\mathrm{QED}_{3}$, or $\mathrm{QED}_{3}$ in short,

$$
\mathcal{L}_{B}=i \bar{\psi} \gamma^{\mu}\left(\partial_{\mu}-i a_{\mu}\right) \psi-\frac{1}{4 \pi} \epsilon^{\mu \nu \lambda} A_{\mu} \partial_{\nu} a_{\lambda}
$$

(A more accurate version of $\mathcal{L}_{B}$ will be given later.) It is usually claimed that the duality between the two theories (35) and (36) implies the CF theory of the half-filled Landau level. The argument runs as follows: A finite magnetic field in free Dirac fermion theory corresponds to a finite density in $\mathrm{QED}_{3}$, and at finite density, the fermions of $\mathrm{QED}_{3}$ form a Fermi liquid. This line of argument, however, hides an important subtle point. For a free fermion in an external magnetic field, the ground state is exponentially degenerate. If the conjectured duality is valid, then $\mathrm{QED}_{3}$ must have finite entropy at finite density - a very unusual situation in quantum field theory indeed, which does not have a direct relationship with the quantum Hall state at $\nu=1 / 2$. What is implicitly assumed here is that small interactions in theory A make the ground state in theory B unique and Fermi-liquid like. In particular, the Fermi velocity of the $\mathrm{CF}$ is determined by terms not present in the basic Lagrangians (35) and (36). One recognizes here the energy scale problem in a different guise.

Another subtlety of the duality is the stability of the theory (36). The question is related to the question about the fate of scale invariance in a theory of $N_{f}$ flavors of fermions coupled to a gauge field. At large $N_{f}$ the theory is conformal; however, it is not clear what is the situation at small $N_{f}$. Approaches based on the gap equation (unreliable because they rely on uncontrolled truncations of the Schwinger-Dyson equation) give rather high values of $N_{f}^{\text {crit }}$; however a lattice simulation [49] implies that the theory remains conformal down to $N_{f}=2$.

One promising approach to derive the duality is the "wire construction" by Alicea, Mross \& Motrunich [50]. Here one discretizes the Dirac fermion theory into a set of $(1+1)$ dimensional fermions and then applies the well-developed machinery of bosonization to these theories. One then applies a linear nonlocal transformations on the bosons and refermionizes the resulting theory. The result is a discretized version of the CF coupled to a gauge field. This approach provides the most explicit mapping of fields between the two sides of the duality. The existence of the continuum limit is assumed, and the approach breaks rotational invariance and continuous translational invariance.

In the rest of the section we will describe a promising approach pioneered in recent works $[51,52]$. This approach is particularly interesting since it places the quantum Hall problem in the context of a large "web of dualities" between different (2+1)-dimensional gauge theories.

The duality between free Dirac fermion and parity-invariant $\mathrm{QED}_{3}$ is similar to the more familiar duality between the a complex scalar field theory near the Wilson-Fisher fixed point (Wilson-Fisher scalar) and that of a scalar field coupled to a U(1) gauge field near the phase transition between the Coulomb phase and the Higgs phase. One of the most important recent insights has been the understanding that the two dualities are consequences of a perhaps more elementary, seed duality. The latter duality is a relativistic version of flux 
attachment.

In this section, the following shortcut notation is used: $a d a \equiv \epsilon^{\mu \nu \lambda} a_{\mu} \partial_{\nu} a_{\lambda}, A d a \equiv$ $\epsilon^{\mu \nu \lambda} A_{\mu} \partial_{\nu} a_{\lambda}$. Moreover, capital letters $(A)$ refer to background gauge fields, lower-case letters $(a, b$, etc.) refer to dynamical gauge fields. The seed duality is between a relativistic bosonic theory coupled to a CS gauge field $a$, and a relativistic fermionic theory,

$$
\mathcal{L}(\phi, a)+\frac{1}{4 \pi} a d a+\frac{1}{2 \pi} A d a \Leftrightarrow \mathcal{L}(\psi, A)-\frac{1}{2} \frac{1}{4 \pi} A d A
$$

Here $\mathcal{L}(\phi, a)$ includes all terms consistent with symmetry, including the mass term $m^{2}|\phi|^{2}$ whose coefficient needs to be tuned to a critical point, and a $|\phi|^{4}$ term whose coefficient is assumed to flow, in the infrared, to a Wilson-Fisher-like fixed point. On the right-hand side is the theory $\mathcal{L}(\psi, A)$, which involves a two-component fermion coupled to the external field $A$. Without the CS term $A d A$, the right-hand side is parity and time-reversal invariant when the mass of the fermion is zero. The statement of the duality is that if one takes the path integral of the exponent of the actions on both side over all fields except the background fields (i.e., $\phi$ and $a$ on the left-hand side and $\psi$ on the right-hand side), one gets the same functional of the external field $A$.

The duality has not been proven but can be checked in the massive limits. In particular

- When the $\phi$ field is massive (with positive $m^{2}$ ), one can integrate out $\phi$ to get nothing. The field $a$ can now be integrated out, and one gets, on the bosonic side, $-\frac{1}{4 \pi} A d A$. On the fermionic side, this corresponds to a positive sign of the mass of the fermion. Integrate out the fermion, one also gets $-\frac{1}{4 \pi} A d A$.

- When $\phi$ condenses (negative $m^{2}$ ), a becomes massive. Integrating out a now gives nothing. On the fermionic side, the fermion mass is negative. Integrating out the fermion, then one cancels out the $A d A$ CS term.

From the seed duality whole web of different dualities can be derived in an almost mechanical manner. We present here a few illustrative cases. If one gauges the gauge field $A$, replacing $A \rightarrow b$, and added a term $\frac{1}{2 \pi} A d b$, with $A$ being a new background field and $b$ a field to be integrated over, one gets the following duality:

$$
\mathcal{L}(\phi, a)+\frac{1}{4 \pi} a d a+\frac{1}{2 \pi} b d a+\frac{1}{2 \pi} A d b \Leftrightarrow \mathcal{L}(\psi, b)-\frac{1}{2} \frac{1}{4 \pi} b d b+\frac{1}{2 \pi} A d b .
$$

On the left-hand side, one can integrate over $b$ and then $a$ to obtain a new duality:

$$
\mathcal{L}(\phi, A)+\frac{1}{4 \pi} A d A \Leftrightarrow \mathcal{L}(\psi, a)-\frac{1}{2} \frac{1}{4 \pi} a d a+\frac{1}{2 \pi} A d a .
$$

This can be interpreted as the statement that a fermion with attached flux is a boson. Note that the right-hand side is gauge invariant: the CS term with half-integer coefficient can be thought of as coming from the Pauli-Villars regularization of $\mathcal{L}(\psi, a)$ when the Pauli-Villars mass goes to infinity. 
The bosonic particle-vortex duality can now be derived from the boson-fermion dualities by a series of formal manipulations. We start from the duality (39), move the AdA term from the left-hand side to the right-hand side, and promote $A$ to a dynamical gauge field as done before. We find

$$
\mathcal{L}(\phi, b)+\frac{1}{2 \pi} A d b \Leftrightarrow \mathcal{L}(\psi, a)-\frac{1}{2} \frac{1}{4 \pi} a d a+\frac{1}{2 \pi} b d a-\frac{1}{4 \pi} b d b+\frac{1}{2 \pi} A d b .
$$

The integral over $b$ on the right-hand side is a Gaussian integral, with the saddle point at $b=a+A$. We find after the integration

$$
\mathcal{L}(\phi, b)+\frac{1}{2 \pi} A d b \Leftrightarrow \mathcal{L}(\psi, a)+\frac{1}{2} \frac{1}{4 \pi} a d a+\frac{1}{2 \pi} A d a+\frac{1}{4 \pi} A d A .
$$

Using the time-reversed version Eq. (39) and applying charge conjugation, we find

$$
\mathcal{L}(\phi, a)+\frac{1}{2 \pi} A d a \Leftrightarrow \mathcal{L}(\tilde{\phi}, A),
$$

which is the well known bosonic particle-vortex duality [35,36]. Although the duality is well known, the success in deriving it gives one some confidence of the correctness of the original seed duality.

A similar set of manipulations lead to the fermionic particle-vortex duality, we start from the duality (39), moving the $A d A$ term to the right-hand side,

$$
\mathcal{L}(\Phi, A) \Leftrightarrow \mathcal{L}(\psi, a)-\frac{1}{2} \frac{1}{4 \pi} a d a+\frac{1}{2 \pi} A d a-\frac{1}{4 \pi} A d A .
$$

Now we gauge $A$ on both sides $(A \rightarrow b)$, but add to the action $-\frac{1}{4 \pi} b d b+\frac{1}{2 \pi} A d b$ before integrating over $b$,

$$
\mathcal{L}(\phi, b)-\frac{1}{4 \pi} b d b+\frac{1}{2 \pi} A d b \Leftrightarrow \mathcal{L}(\psi, a)-\frac{1}{2} \frac{1}{4 \pi} a d a+\frac{1}{2 \pi} b d a-\frac{2}{4 \pi} b d b+\frac{1}{2 \pi} A d b .
$$

Using the time-reversed version of Eq. (37) one can transform the left-hand side to a fermionic theory. After moving the terms $\operatorname{Ad} A$ between sides, one finds at the end

$$
\mathcal{L}(\tilde{\psi}, A) \Leftrightarrow \mathcal{L}(\psi, a)-\frac{1}{2} \frac{1}{4 \pi} a d a+\frac{1}{2 \pi} b d a-\frac{2}{4 \pi} b d b+\frac{1}{2 \pi} A d b-\frac{1}{2} \frac{1}{4 \pi} A d A .
$$

This is more accurate version of the fermionic particle-vortex duality that would underlie the CF picture of the half-flled Landau level. The previous, naïve version of the duality is obtained by trying to integrate over $a$. The saddle point equation for this equation is

$$
d b-2 d a+d A=0,
$$

and if one sets $a=(b+A) / 2$ (which in general violates the quantization condition) one obtains the fermionic duality mentioned above. The fact that the right-hand side of Eq. (45) is a time-reversal symmetric theory is not obvious, but can be demonstrated [52].

A whole web of dualities can be derived from the basic boson-fermion duality in a manner similar to the one we have used above. A full account of these duality is beyond the scope of this review. Some remarkable insights obtained in this direction include a self-dual theory of Ref. [53] and various descriptions of a possible $\mathrm{SO}(5)$ quantum critical point [54]. 


\section{CONCLUSION}

We have presented arguments in favor of the Dirac nature of the CF. The Dirac CF provides a very simple solution to a number of puzzles that have been plaguing the field theory of the CF for a long time. Regarded as a low-energy effective theory, the Dirac CF theory, modified to include the electric dipole moment of the $\mathrm{CF}$, provides a description consistent with low-energy constraints.

We have also reviewed various physical consequences of the Dirac CF theory. While most features of the HLR theory is preserved by the Dirac CF theory, the latter leads to distinct physical consequences, which are only started to be explored experimentally [55].

\section{DISCLOSURE STATEMENT}

The author is not aware of any affiliations, membership, funding, or financial holdings that might be perceived as affecting the objectivity of this review.

\section{ACKNOWLEDGMENTS}

This work is supported, in part, by US Department Of Energy grant No. DE-FG02-13ER41958, the Army Research Office-Multidisciplinary University Research Initiative grant No. 63834PH-MUR, and a Simons Investigator Grant from the Simons Foundation. Additional support was provided by the Chicago Materials Research Science \& Engineering Center, which is funded by the National Science Foundation through grant DMR-1420709.

\section{LITERATURE CITED}

[1] Tsui DC, Stormer HL, Gossard AC. 1982. Phys. Rev. Lett. 48:1559-62

[2] Laughlin RB. 1983. Phys. Rev. Lett. 50:1395-98

[3] Halperin BI, Lee PA, Read N. 1993. Phys. Rev. B 47:7312-43

[4] Son DT. 2015. Phys. Rev. X 5:031027

[5] Arovas DP, Schrieffer JR, Wilczek F, Zee A. 1985. Nucl. Phys. B 251:117-26

[6] Zhang SC, Hansson TH, Kivelson S. 1988. Phys. Rev. Lett. 62:82-85

[7] Jain JK. 1989. Phys. Rev. Lett. 63:199-202

[8] Lopez A, Fradkin E. 1991. Phys. Rev. B 44:5246-62

[9] Willett RL, Paalanen MA, Ruel RR, West KW, Pfeiffer LN, Bishop DJ. 1990. Phys. Rev. Lett. 65:112-15

[10] Kang W, Stormer HL, Pfeiffer LN, Baldwin KW, West KW. 1993. Phys. Rev. Lett. $71: 3850-53$

[11] Goldman VJ, Su B, Jain JK. 1994. Phys. Rev. Lett. 72:2065-68

[12] Shankar R, Murthy G. 1997. Phys. Rev. Lett. 79:4437-40 
[13] Pasquier V, Haldane FDM. 1998. Nucl. Phys. B 516:719-26

[14] Read N. 1998. Phys. Rev. B 58:16262-90

[15] Levin M, Son DT. 2017. Phys. Rev. B 95:125120

[16] Son DT. 2013. arXiv:1306.0638

[17] Jensen K. 2014. arXiv:hep-th/1408.6855

[18] Geracie M, Prabhu K, Roberts MM. 2015a. J. High Energy Phys. 08:042

[19] Geracie M, Prabhu K, Roberts MM. 2015b. J. Math. Phys. 56:103505

[20] Moore GW, Read N. 1991. Nucl. Phys. B360:362-96

[21] Girvin SM. 1984. Phys. Rev. B 29:6012-14

[22] Wang C, Cooper NR, Halperin BI, Stern A. 2017a. Phys. Rev. X 7:031029

[23] Golkar S, Nguyen DX, Roberts MM, Son DT. 2016. Phys. Rev. Lett. 117:216403

[24] Kivelson SA, Lee DH, Krotov Y, Gan J. 1997. Phys. Rev. B 55:15552-61

[25] Nguyen DX, Golkar S, Roberts MM, Son DT. 2017. arXiv:1709.07885

[26] You Y, Cho GY, Fradkin E. 2016. Phys. Rev. B 93:205401

[27] Barkeshli M, Mulligan M, Fisher MPA. 2015. Phys. Rev. B 92:165125

[28] Levin M, Halperin BI, Rosenow B. 2007. Phys. Rev. Lett. 99:236806

[29] Lee SS, Ryu S, Nayak C, Fisher MPA. 2007. Phys. Rev. Lett 99:236807

[30] Kamburov D, Liu Y, Mueed MA, Shayegan M, Pfeiffer LN, et al. 2014. Phys. Rev. Lett 113:196801

[31] Geraedts SD, Zaletel MP, Mong RSK, Metlitski MA, Vishwanath A, Motrunich OI. 2015. Science 352:197-201

[32] Novoselov KS, Geim AK, Morozov SV, Jiang D, Katsnelson MI, et al. 2005. Nature 438:197-200

[33] Zhang Y, Tan YW, Stormer HL, Kim P. 2005. Nature 438:201-4

[34] Levin M, Son DT. 2015. unpublished

[35] Peskin ME. 1978. Ann. Phys. (N.Y.) 113:122-52

[36] Dasgupta C, Halperin BI. 1981. Phys. Rev. Lett. 47:1556-1560

[37] Haldane FDM. 2004. Phys. Rev. Lett. 93:206602

[38] Read N. 1994. Semicond. Sci. Technol. 9:1859-64

[39] Read N. 1996. Surface Sci. 361/362:7-12

[40] Wang C, Senthil T. 2016. Phys. Rev. B 93:085110

[41] Potter AC, Serbyn M, Vishwanath A. 2016. Phys. Rev. X 6:031026

[42] Wang C, Potter AC, Senthil T. 2013. Phys. Rev. B 88:115137

[43] Bonderson P, Nayak C, Qi XL. 2013. J. Stat. Mech. 2013:P09016

[44] Chen X, Fidkowski L, Vishwanath A. 2014. Phys. Rev. B 89:165132

[45] Metlitski MA, Kane CL, Fisher MPA. 2015. Phys. Rev. B 92:125111

[46] Yang J. 2017. arXiv:1701.03562

[47] Zucker PT, Feldman DE. 2016. Phys. Rev. Lett. 117:096802

[48] Murthy G, Shankar R. 2016. Phys. Rev. B 93:085405

[49] Karthik N, Narayanan R. 2016. Phys. Rev. D93:045020 
[50] Mross DF, Alicea J, Motrunich OI. 2016. Phys. Rev. Lett. 117:016802

[51] Karch A, Tong D. 2016. Phys. Rev. X 6:031043

[52] Seiberg N, Senthil T, Wang C, Witten E. 2016. Ann. Phys. 374:395-433

[53] Xu C, You YZ. 2015. Phys. Rev. B 92:220416

[54] Wang C, Nahum A, Metlitski MA, Xu C, Senthil T. 2017b. Phys. Rev. X 7:031051

[55] Pan W, Kang W, Baldwin KW, West KW, Pfeiffer LN, Tsui DC. 2017. Nat. Phys. $13: 1168-72$ 